\title{
A Perturbative Stochastic Galerkin Method for the Uncertainty Quantification of Linear Circuits
}

\author{
Paolo Manfredi, Senior Member, IEEE, Riccardo Trinchero, Member, IEEE, Dries Vande Ginste, Senior \\ Member, IEEE
}

\begin{abstract}
This paper presents an iterative and decoupled perturbative stochastic Galerkin (SG) method for the variability analysis of stochastic linear circuits with a large number of uncertain parameters. State-of-the-art implementations of polynomial chaos expansion and SG projection produce a large deterministic circuit that is fully coupled, thus becoming cumbersome to implement and inefficient to solve when the number of random parameters is large. In a perturbative approach, component variability is interpreted as a perturbation of its nominal value. The relaxation of the resulting equations and the application of a SG method lead to a decoupled system of equations, corresponding to a modified equivalent circuit in which each stochastic component is replaced by the nominal element equipped with a parallel current source accounting for the effect of variability. The solution of the perturbation problem is carried out in an iterative manner by suitably updating the equivalent current sources by means of Jacobi- or Gauss-Seidel strategies, until convergence is reached. A sparse implementation allows avoiding the refinement of negligible coefficients, yielding further efficiency improvement. Moreover, for time-invariant circuits, the iterations are effectively performed in post-processing after characterizing the circuit in time or frequency domain by means of a limited number of simulations. Several application examples are used to illustrate the proposed technique and highlight its performance and computational advantages.
\end{abstract}

Index Terms-Circuit modeling, circuit simulation, perturbation method, polynomial chaos, stochastic Galerkin method, stochastic circuits, tolerance analysis, uncertainty quantification, variability analysis.

\section{INTRODUCTION}

$\mathbf{U}$ NCERTAINTY quantification of electrical and electronic circuits became of paramount importance to account for process variations and uncontrollable operating conditions in modern mass-production scenarios. While virtually all commercial circuit simulators implement the brute-force Monte Carlo (MC) method for variability analysis, it is well know that it suffers from poor convergence properties [1], thus rapidly becoming intractable for the accurate characterization of a realistic design.

In the last decade, polynomial chaos expansion (PCE) was extensively investigated and leveraged as a powerful alternative tool for uncertainty quantification in many engineering domains, including electronics [2]. PCE-based methods comprise a rather broad class of approaches that are based on expanding

P. Manfredi and R. Trinchero are with the EMC Group, Department of Electronics and Telecommunications, Politecnico di Torino, 10129 Torino, Italy (e-mail: paolo.manfredi@polito.it, riccardo.trinchero@polito.it).

D. Vande Ginste is with the Electromagnetics Group, Department of Information Technology, Ghent University-imec, 9000 Gent, Belgium (e-mail: dries.vandeginste@ugent.be). stochastic quantities of interest in terms of polynomials that are orthogonal with respect to the probability density function of the uncertain parameters [3]. The different implementations can be grouped into two main categories: intrusive and nonintrusive methods [4].

The main intrusive approach is the so-called stochastic Galerkin (SG) method [5]. It consists in replacing stochastic quantities inside the system of governing equations with their respective PCEs, and applying a Galerkin projection to arrive at an augmented system of deterministic equations in the unknown expansion coefficients. It is recognized as the most accurate numerical method for the evaluation of the PCE coefficients [4], [6], but it requires a modification of the equations, which may in turn require the development of a dedicated solver. Applications of the SG method to circuit simulations are found in [7]-[10], where a customized, SPICElike circuit solver was developed to handle the projected equations. In [11] and [12], SPICE-compatible circuit models were introduced for the Galerkin equations, allowing one to perform the SG simulation in standard SPICE-type solvers by systematically creating a compatible companion network. The passivity of such models was also discussed in an earlier paper [13].

The use of Galerkin projection limits the method to linear systems or systems with polynomial nonlinearities [14]. Alternative numerical strategies are required to embed generic nonlinear circuit components [12], [15], [16]. Moreover, since the resulting SG-augmented system is large and fully coupled, it often becomes time and memory inefficient to simulate when the number of random parameters is increased.

Another intrusive technique is the stochastic testing (ST) method [17], which is based on point-matching the equations in the stochastic space and internally decoupling them. It also requires a customized solver to handle the modified equations. A non-intrusive reformulation of the ST method was proposed in [18], which recasts the technique as an interpolation in the random space and allows for efficient handling of large numbers of random parameters. A similar technique is the sparse linear regression proposed in [19]. However, interpolation strategies can lead to large errors, in particular for some examples characterized by a large sensitivity of the response with respect to random parameters, as shown later in this paper.

The class of non-intrusive approaches is rather broad and also includes techniques based on least square regression [20], [21], whose accuracy strongly depends on the choice and number of regression samples, as well tensor- 
based approaches [22], which are mostly effective for scalar outputs, while becoming cumbersome to characterize an entire response in time or frequency domain.

This paper focuses on the accurate SG method and proposes an improved reformulation for stochastic linear lumped elements, by recasting it as a perturbation approach, which leads to a decoupled problem that is solved iteratively. The interpretation of the new equations yields an equivalent circuit with the same complexity as the original one, in which however, stochastic components are replaced by a nominal element paralleled by an equivalent current source. Since only the equivalent sources need to be updated throughout the iterations, linear-time invariant (LTI) circuits are effectively characterized in time or frequency domain by means of a limited number of simulations, and the updates are carried out in post-processing, thus providing further computational improvement. Additional efficiency improvement is achieved by adopting a sparse implementation that avoids the refinement of negligible coefficients. Compared to the standard SG implementation for high-dimensional problems, the advocated method scales more favorably with the number of PCE coefficients, and it is compatible with general circuit solvers. Furthermore, as the number of required iterations depends on the amount of variability in the circuit parameters, the method is most efficient for problems with a moderate uncertainty of the circuit components.

Preliminary work on this topic was presented in [23] in which, however, only a model for stochastic resistors was presented. The present paper instead:

- covers the theory in much more detail;

- introduces models for generic linear elements and, specifically, for inductors and capacitors;

- discusses two different strategies to update the equivalent sources, highlighting their pros and cons;

- introduces a sparse implementation that avoids the unnecessary refinement of negligible coefficients;

- introduces a more efficient implementation for LTI circuits based on time- or frequency-domain characterization;

- provides more extensive validation examples and a comprehensive performance analysis.

In particular, the proposed method is illustrated by means of several linear circuits, including filters (both passive and active), a network with delay elements, and a switching converter.

The remainder of the paper is organized as follows. Section II provides the necessary basic notions about the PCE and the SG method. In Section III, the proposed perturbative SG method and the corresponding equivalent circuit models are presented. Section IV discusses two strategies to update the equivalent sources accounting for the component variability, as well as a sparse implementation thereof. A more efficient implementation for LTI circuits is introduced in Section V. Extensive validation examples are provided in Section VI, and the performance is carefully assessed and compared against state-of-the-art techniques in Section VII. Finally, conclusions are drawn in Section VIII.

\section{Polynomial Chaos Overview}

This section reviews the necessary notions about state-ofthe-art polynomial chaos implementations, as needed in the subsequent developments. Consider a circuit with $d$ uncertain elements, whose variability is parameterized by a vector of normalized random variables $\boldsymbol{\xi}=\left(\xi_{1}, \ldots, \xi_{d}\right)$.

\section{A. Polynomial Chaos Expansion}

Any stochastic voltage or current in the circuit, generically denoted with $x$, is approximated by a truncated PCE [3], i.e.,

$$
x(t, \boldsymbol{\xi}) \approx \hat{x}(t, \boldsymbol{\xi})=\sum_{k=1}^{K} x_{k}(t) \varphi_{k}(\boldsymbol{\xi}) .
$$

The basis functions $\varphi_{k}$ are orthonormal with respect to the inner product

$$
\langle f, g\rangle=\int_{\mathbb{R}^{d}} f(\boldsymbol{\xi}) g(\boldsymbol{\xi}) \rho(\boldsymbol{\xi}) d \boldsymbol{\xi},
$$

where $\rho(\boldsymbol{\xi})$ is the joint probability density function (PDF) of the random variables $\xi$. For the univariate case, i.e., $d=1$, and standard distributions, orthogonal polynomials are readily available. For example, Hermite, Legendre, and Jacobi polynomials are orthogonal when the distribution $\rho(\xi)$ is Gaussian, uniform, or beta, respectively. Normalization of the basis functions is useful and readily achieved by properly rescaling the polynomials.

For multiple and independent uncertain parameters, suitable basis functions are constructed as a tensor-product combination of univariate polynomials in each variable:

$$
\varphi_{k}(\boldsymbol{\xi})=\prod_{j=1}^{d} \phi_{k_{j}}\left(\xi_{j}\right),
$$

where $\phi_{k_{j}}$ is a polynomial of degree $k_{j}$. For non-standard distributions, including dependent or correlated ones, suitable orthonormal polynomials can be numerically constructed using a Gram-Schmidt orthogonalization [24] or Cholesky decomposition [25].

In (3), $k$ is a positive integer that maps to a vectorial multi-index element $\boldsymbol{k}=\left(k_{1}, \ldots, k_{d}\right) \in \mathcal{K}$. The expansion is typically truncated to retain all basis functions up to a total degree $p$, meaning that the set of multi-indices is defined as

$$
\mathcal{K}=\left\{\boldsymbol{k}:\|\boldsymbol{k}\|_{1} \leq p\right\},
$$

and its cardinality is $K=|\mathcal{K}|=(p+d) ! /(p ! d !)$.

The basis functions are further assumed to be sorted in ascending graded lexicographic order [26]. Under this assumption, the first two statistical moments (i.e., mean and variance) of the approximated quantity in (1) are readily given by

$$
\mathrm{E}\{x(t, \boldsymbol{\xi})\} \approx \mathrm{E}\{\hat{x}(t, \boldsymbol{\xi})\}=x_{1}(t)
$$

and

$$
\operatorname{Var}\{x(t, \boldsymbol{\xi})\} \approx \operatorname{Var}\{\hat{x}(t, \boldsymbol{\xi})\}=\sum_{k=2}^{K} x_{k}^{2}(t) .
$$

Other moments, as well as distribution functions, are estimated by randomly sampling (1). 


\section{B. Non-Intrusive Techniques}

There exist many different approaches for the determination of the PCE coefficients in (1). Among non-intrusive methods, regression-based approaches fit the PCE in a least-square sense [20], [21], but they often require a rather large number of simulation samples $(\gg K$, typically at least $2 K$ ) for the regression problem to be sufficiently overdetermined, and hence the solution accurate. Interpolation-based techniques [18], [19] require $K$ simulation samples, but the accuracy is not always guaranteed, as will be shown by the proposed application examples, and it is generally lower than with Galerkin-based methods [27]. Other techniques based on Gaussian quadratures [4] also require large sample sizes and were proven to be, under certain conditions, an approximation of the SG method [28].

\section{SG Method}

While all the aforementioned approaches do not require explicit access to the system equations, the SG method [5] is an intrusive approach which is however recognized as the most accurate strategy for the evaluation of the PCE coefficients [4], [6], [27]. In [12], an element-wise approach for stochastic circuits was presented, where the governing equations of uncertain linear lumped elements were expanded and Galerkinprojected to arrive at deterministic and augmented systems of equations. The approach is summarized below.

Consider a generic equation for a linear component of the form

$$
x(t, \boldsymbol{\xi})=\mathcal{X}(\boldsymbol{\xi}) \frac{d^{\alpha}}{d t^{\alpha}} y(t, \boldsymbol{\xi})
$$

where $\alpha=0$ for static elements (e.g., resistors) and $\alpha=1$ for dynamic elements (inductors and capacitors), $x, y$ denote a generic current or voltage variable, and $\mathcal{X}$ is the pertinent element value. Replacing each stochastic (i.e., $\boldsymbol{\xi}$-dependent) quantity in (7) with its corresponding PCE (1) leads to

$$
\sum_{k=1}^{K} x_{k}(t) \varphi_{k}(\boldsymbol{\xi})=\sum_{k=1}^{K} \sum_{j=1}^{K} \mathcal{X}_{k} \frac{d^{\alpha}}{d t^{\alpha}} y_{j}(t) \varphi_{k}(\boldsymbol{\xi}) \varphi_{j}(\boldsymbol{\xi})
$$

A Galerkin testing procedure, i.e., projecting the above equation onto the $K$ basis functions using the inner product (2), allows obtaining $K$ coupled and deterministic equations for each PCE coefficient:

$$
x_{k}(t)=\sum_{j=1}^{K} \widetilde{\mathcal{X}}_{k j} \frac{d^{\alpha}}{d t^{\alpha}} y_{j}(t), \quad \forall k=1, \ldots, K
$$

where

$$
\widetilde{\mathcal{X}}_{k j}=\sum_{i=1}^{K} \mathcal{X}_{i}\left\langle\varphi_{k} \varphi_{j}, \varphi_{i}\right\rangle
$$

The triple product term $\left\langle\varphi_{k} \varphi_{j}, \varphi_{i}\right\rangle$ is analytically available for standard polynomials. Anyhow, the values can be computed a-priori up to a predefined maximum order and stored into look-up tables.

In [12], the SG equations (9) for resistors, inductors, and capacitors were interpreted as equivalent circuits compatible with (advanced) SPICE-type simulators. Eventually, a deterministic and augmented counterpart of the original stochastic network was systematically created by connecting the models for stochastic and non-stochastic elements. The nodal voltages and branch currents of the new companion network corresponded to the PCE coefficients of those in the original circuit. Therefore, a single deterministic simulation of this equivalent circuit, carried out in a standard circuit solver, allowed retrieving the sought-after PCE coefficients and performing uncertainty quantification.

\section{Limitations of the SG Method}

Albeit very accurate, the SG method suffers from some limitations. First of all, a new, augmented circuit needs to be constructed. This is relatively simple but, in order to account for all coupling in (9), extensive use is made of behavioral controlled sources. Depending on the specific component type and circuit simulator environment, the implementation of (9) becomes cumbersome. Non-stochastic components must be suitably incorporated in the augmented network, and this is not always straightforward. Furthermore, the CPU time has typically superlinear scaling because of the coupling. In the next section, a new perturbative approach is put forward that helps mitigate both the aforementioned inconveniences.

\section{Perturbative SG Method}

This section introduces a perturbative and iterative SG method for the simulation of linear circuits. Although the same rationale can be applied to the global system of equations describing the entire circuit, an element-level approach is adopted here, in line with [12], thus providing a modular technique that can be easily integrated into standard commercial simulators.

\section{A. Resistor}

The proposed method is first introduced for a stochastic resistor, whose governing equation reads

$$
v(t, \boldsymbol{\xi})=R(\boldsymbol{\xi}) i(t, \boldsymbol{\xi})
$$

with obvious variable definitions. The value of the stochastic resistance is expressed as a random deviation from a deterministic nominal value, i.e., $R(\boldsymbol{\xi})=\bar{R}+\mathcal{R}(\boldsymbol{\xi})$. Under the assumption that $\mathcal{R}(\boldsymbol{\xi}) \ll \bar{R}$ (the proposed application examples will help quantify this condition), replacing the above expression into (11) and applying a relaxation leads to

$$
v^{(m)}(t, \boldsymbol{\xi})=\bar{R} i^{(m)}(t, \boldsymbol{\xi})+\mathcal{R}(\boldsymbol{\xi}) i^{(m-1)}(t, \boldsymbol{\xi})
$$

where the superscript $m \geq 0$ denotes the iteration index, and $i^{(-1)}=0$. Application of the SG projection yields a set of $K$ equations

$$
v_{k}^{(m)}(t)=\bar{R} i_{k}^{(m)}(t)+\underbrace{\sum_{j=1}^{K} \widetilde{\mathcal{R}}_{k j} i_{j}^{(m-1)}(t)}_{v_{e q, k}^{(m)}(t)},
$$

where $v_{k}^{(m)}$ and $i_{k}^{(m)}$ denote the PCE coefficients of the resistor voltage and current at the $m$ th iteration, respectively, whereas 
the coefficients $\widetilde{\mathcal{R}}_{k j}$ are constructed as in (10). It is noted that the second term in the right-hand side of (13) depends on the PCE coefficients of the current at the previous iteration, and it is therefore known at any given iteration. As such, it plays the role of an equivalent independent voltage source $v_{e q, k}$ in series with the resistor.

Since SPICE-base simulators mainly solve the circuit for nodal voltages, a voltage-driven model is preferred to avoid the introduction of additional nodes. Therefore, (13) is rewritten as

$$
i_{k}^{(m)}(t)=\frac{1}{\bar{R}} v_{k}^{(m)}(t) \underbrace{-\sum_{j=1}^{K} \frac{\widetilde{\mathcal{R}}_{k j}}{\bar{R}} i_{j}^{(m-1)}(t)}_{i_{e q, k}^{(m)}(t)},
$$

which corresponds to a resistor with resistance $\bar{R}$ paralleled by an independent current source $i_{e q, k}$.

It is important to point out that, thanks to the relaxation, the equations (14) are uncoupled, unlike (9), as the coupling between the PCE coefficients is translated into known equivalent sources. The value of the independent sources in (14) differs for each PCE coefficient that is solved for, and it is updated throughout the iterations in a way that is discussed in detail in Section IV.

\section{B. Inductor}

Similarly, the equation of a stochastic inductor

$$
v(t, \boldsymbol{\xi})=L(\boldsymbol{\xi}) \frac{d}{d t} i(t, \boldsymbol{\xi}),
$$

with $L(\boldsymbol{\xi})=\bar{L}+\mathcal{L}(\boldsymbol{\xi})$, is relaxed as

$$
v^{(m)}(t, \boldsymbol{\xi})=\bar{L} i^{(m)}(t, \boldsymbol{\xi})+\mathcal{L}(\boldsymbol{\xi}) \frac{d}{d t} i^{(m-1)}(t, \boldsymbol{\xi}) .
$$

Application of the SG projection leads to

$$
v_{k}^{(m)}(t)=\bar{L} \frac{d}{d t} i_{k}^{(m)}(t)+\underbrace{\sum_{j=1}^{K} \widetilde{\mathcal{L}}_{k j} \frac{d}{d t} i_{j}^{(m-1)}(t)}_{v_{e q, k}(t)}
$$

which, by inverting the current-voltage relationship, is recast in voltage-driven form as

$$
i_{k}^{(m)}(t)=\frac{1}{\bar{L}} \int_{-\infty}^{t} v_{k}^{(m)}(t) d t \underbrace{-\sum_{j=1}^{K} \frac{\widetilde{\mathcal{L}}_{k j}}{\bar{L}} i_{k}^{(m-1)}(t)}_{i_{e q, k}(t)}
$$

The above equation corresponds to an inductor of inductance $\bar{L}$ paralleled by an equivalent current source.

\section{Capacitor}

Finally, the equation of a stochastic capacitor

$$
i(t, \boldsymbol{\xi})=C(\boldsymbol{\xi}) \frac{d}{d t} v(t, \boldsymbol{\xi}),
$$

with $C(\boldsymbol{\xi})=\bar{C}+\mathcal{C}(\boldsymbol{\xi})$, is relaxed as

$$
i^{(m)}(t, \boldsymbol{\xi})=\bar{C} \frac{d}{d t} v^{(m)}(t, \boldsymbol{\xi})+\mathcal{C}(\boldsymbol{\xi}) \frac{d}{d t} v^{(m-1)}(t, \boldsymbol{\xi}) .
$$

Application of the SG projection produces

$$
i_{k}^{(m)}(t)=\bar{C} \frac{d}{d t} v_{k}^{(m)}(t)+\sum_{j=1}^{K} \widetilde{\mathcal{C}}_{k j} \frac{d}{d t} v_{j}^{(m-1)}(t) .
$$

By noting that

$$
\frac{d}{d t} v_{j}^{(m-1)}=\frac{1}{\bar{C}} i_{C, j}^{(m-1)},
$$

with $i_{C, j}^{(m-1)}$ being the current flowing into the capacitor $\bar{C}$ at the previous iteration, the above equation is recast as

$$
i_{k}^{(m)}(t)=\bar{C} \frac{d}{d t} v_{k}^{(m)}(t)+\underbrace{\sum_{j=1}^{K} \frac{\widetilde{\mathcal{C}}_{k j}}{\bar{C}} i_{C, j}^{(m-1)}(t)}_{i_{e q, k}^{(m)}},
$$

which has a similar form as (14) and (18), and avoids differentiations in the evaluation of the equivalent current source $i_{e q, k}$. Similarly to the previous models, the equation corresponds to a capacitor with capacitance $\bar{C}$ paralleled by an independent current source.

The models so far introduced for the basic RLC elements are readily generalized to linear dependent sources with stochastic coupling coefficients.

\section{Independent Sources}

The equivalent sources for stochastic elements in (14), (18), and (23), add to the original independent (voltage or current) sources that provide the actual excitation of the circuit. Variability is commonly assumed on circuit components, whereas independent sources are often treated as deterministic. The generic equation for a deterministic independent source is

$$
x(t, \boldsymbol{\xi})=u(t),
$$

where $x$ and $U$ are either a voltage or a current. Replacing the left-hand side with the corresponding PCE and applying Galerkin projection leads to

$$
x_{k}(t)= \begin{cases}u(t) & k=1 \\ 0 & k>1\end{cases}
$$

Therefore, the original independent sources are retained only when solving for the first PCE coefficients, and set to zero otherwise (cfr. [11], [12]) .

\section{E. Summary and Implementational Details}

Figure 1 summarizes the equivalent circuits for stochastic RLC elements and deterministic independent sources. It should be noted that, for capacitors, the equivalent current source depends on the sole element current with positive sign, whereas for resistors and inductors it depends on the total branch current, taken with negative sign.

Briefly speaking, the modification of the original network amounts to replacing stochastic elements with the nominal component equipped with a parallel independent current source. Deterministic components remain unaffected. As a result, the complexity of the equivalent circuit for the perturbative SG simulation is greatly simplified compared to [12]. 


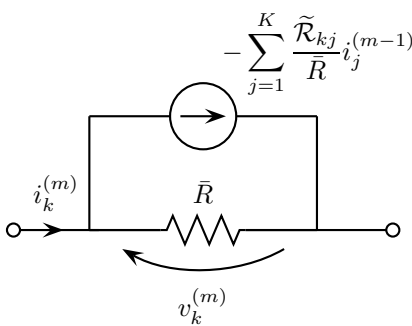

(a)

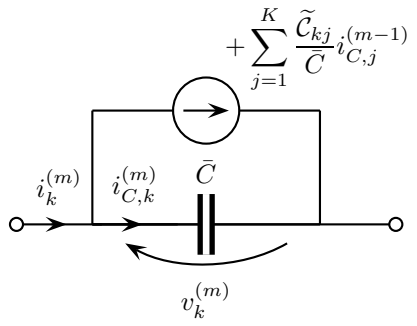

(c)

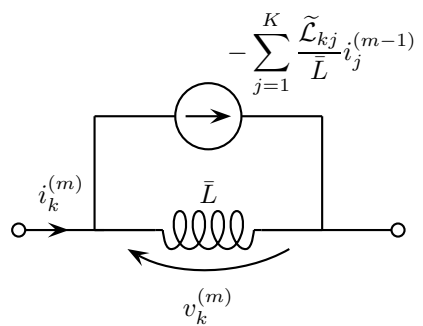

(b)

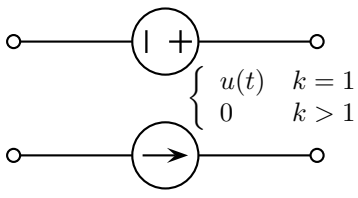

(d)
Fig. 1. Perturbative circuit models for: (a) stochastic resistors; (b) stochastic inductors; (c) stochastic capacitors; (d) deterministic independent sources.

The new circuit is iteratively simulated to retrieve the various PCE coefficients of the stochastic voltages and currents, by updating the equivalent sources of the uncertain elements until convergence is reached.

As already noted for (14), also equations (18) and (23) are uncoupled, as the effect of coupling between the PCE coefficients is taken into account by the equivalent sources. Hence, each PCE coefficient can be solved for individually, either sequentially or possibly in parallel, depending on the strategy for the update of the equivalent sources, which is discussed in Section IV. Throughout the iterations, the equivalent current sources of stochastic elements are suitably updated, whereas the original independent sources are switched off when simulating for the PCE coefficients with $k>1$. At each iteration, a circuit of the same complexity as the original one is simulated, which leads to a computational cost exhibiting a favorable scaling with respect to the SG size factor $K$.

At iteration $m=0$, all equivalent sources are zero. Therefore, the first iteration for $k=1$ is actually equivalent to solving the circuit for the nominal configuration of the components. This simulation thus immediately provides the result of the nominal circuit to the circuit designer without additional cost. As the circuit has no excitation for $m=0$ and $k>1$, the corresponding simulations can be skipped.

\section{Update of the Equivalent CurRent Sources}

The method outlined in Section III resembles the iterative techniques for the solution of the linear system of equations [29]. Borrowing from linear algebra, there are two common strategies that can be used to update the equivalent sources, namely Jacobi and Gauss-Seidel updates. Each one has both advantages and disadvantages, as discussed in the following.

\section{A. Jacobi Update}

According to the Jacobi update, the $K$ equivalent sources for all the PCE coefficients are simultaneously updated at the beginning of each iteration, based on the solutions of the element currents at the previous iteration. The equivalent sources are updated by means of a single matrix-vector multiplication of the form

$$
\boldsymbol{i}_{e q}^{(m)}(t)=\widetilde{\mathcal{X}} i_{u p}^{(m-1)}(t),
$$

where $\boldsymbol{i}_{e q}^{(m)}$ and $\boldsymbol{i}_{u p}^{(m-1)}$ are vectors in $\mathbb{R}^{K}$ collecting all the equivalent sources and all the currents needed for their update, respectively, whereas $\widetilde{\mathcal{X}} \in \mathbb{R}^{K \times K}$ is a (typically sparse) matrix with entries that readily follow from (14), (18), or (23). Since all the equivalent currents are available concurrently, at each iteration the $K$ circuit solutions for the various PCE coefficients can, in principle, be carried out in parallel.

The pseudo-code for the perturbative SG solution with Jacobi updates is provided by Algorithm 1. The main drawback of this strategy is its slower convergence compared to a more efficient alternative, i.e., the Gauss-Seidel update, which is discussed next.

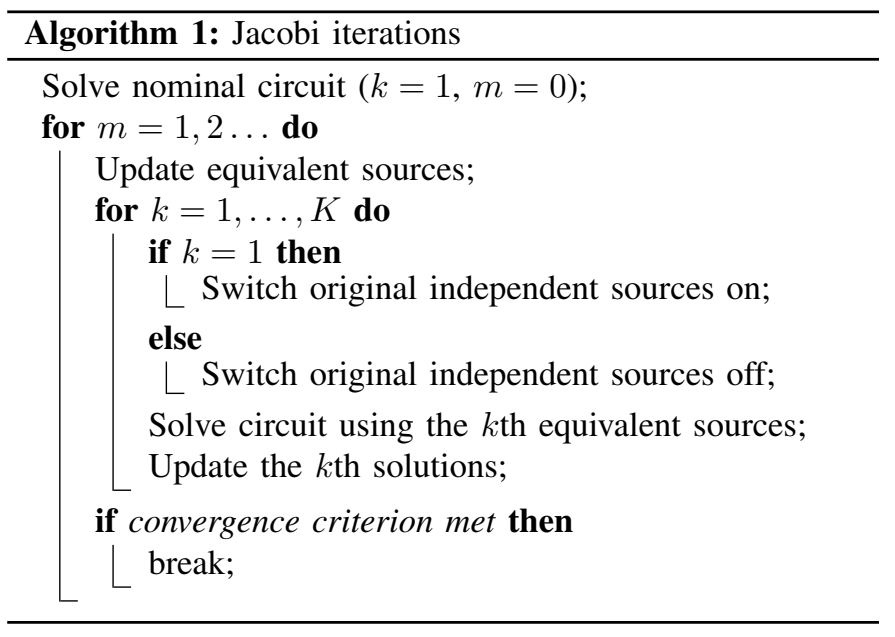

\section{B. Gauss-Seidel Update}

In each iteration $m$, for the update of the $k$ th coefficient, we may also include the information already obtained for the coefficients $1, \ldots, k-1$ during the computation of the equivalent current sources, as follows:

$$
i_{e q, k}^{(m)}(t)=\sum_{j=1}^{k-1} \widetilde{\mathcal{X}}_{k j} i_{u p, j}^{(m)}(t)+\sum_{j=k}^{K} \widetilde{\mathcal{X}}_{k j} i_{u p, j}^{(m-1)}(t),
$$

with $\widetilde{\mathcal{X}}_{k j}$ being the entries of the same matrix $\widetilde{\mathcal{X}}$ as in (26). In practice, it suffices to update the equivalent sources inside the inner loop by multiplying the $k$ th row of matrix $\widetilde{\mathcal{X}}$ by the updated vector of the currents, instead of pre-computing all of them at the beginning of the outer loop. The pseudo-code is provided by Algorithm 2.

This is a Gauss-Seidel approach, in which the equivalent sources are updated sequentially inside the inner loop for $k$, by exploiting the solutions that have already been computed at a 
given iteration [32]. Briefly speaking, the available information is used immediately, rather than waiting for the next iteration, thus introducing a substantial speed-up in the convergence. The number of iterations is reduced roughly by a factor two, as will be shown by the application examples. Despite the obvious advantage of reducing the number of iterations, a trade-off exists between Jacobi and Gauss-Seidel updates in the efficiency of the calculations, as will be discussed in Sections V and VII.

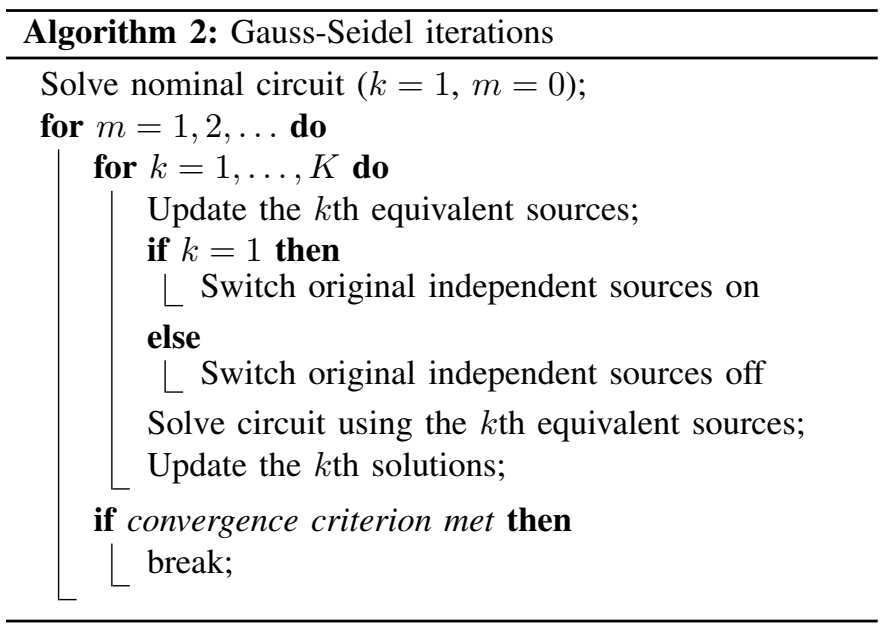

\section{Sparse Implementation}

It is known that PCEs are characterized by the principle of sparsity of effects, meaning that most coefficients in a high-dimensional expansion are negligible [22], [30], [31]. As opposed to the conventional SG implementation, which simultaneously solves for all coefficients, the proposed perturbative implementation can exploit this property by avoiding the refinement of PCE coefficients that are detected to be smaller than a prescribed tolerance. In this paper, a PCE coefficient is considered to be negligible if, after the first iteration, its maximum value over time (or frequency) is below a certain fraction of the maximum value over time (or frequency) of the nominal solution, i.e.,

$$
\max _{t}\left\{x_{k}^{(m)}(t)\right\}<\delta \cdot \max _{t}\left\{x_{1}^{(0)}(t)\right\}
$$

This choice offers a reasonable criterion to identify sparse coefficients at runtime. In the following examples, a threshold of $\delta=10^{-3}$ is considered to assess sparsity.

\section{Summary of Workflow}

Figure 2 illustrates the workflow for the simulation of an RLC circuit. The topology is depicted in the upper box, and all three RLC elements are stochastic. After performing the solution of the nominal circuit, the circuit is re-simulated for each of the $K$ PCE coefficients by including the corresponding equivalent sources (shown in red). The original voltage source is displayed with dashed lines because it is activated only when simulating for $k=1$. Depending on whether Gauss-Seidel or Jacobi updates are used, the sources are updated inside the

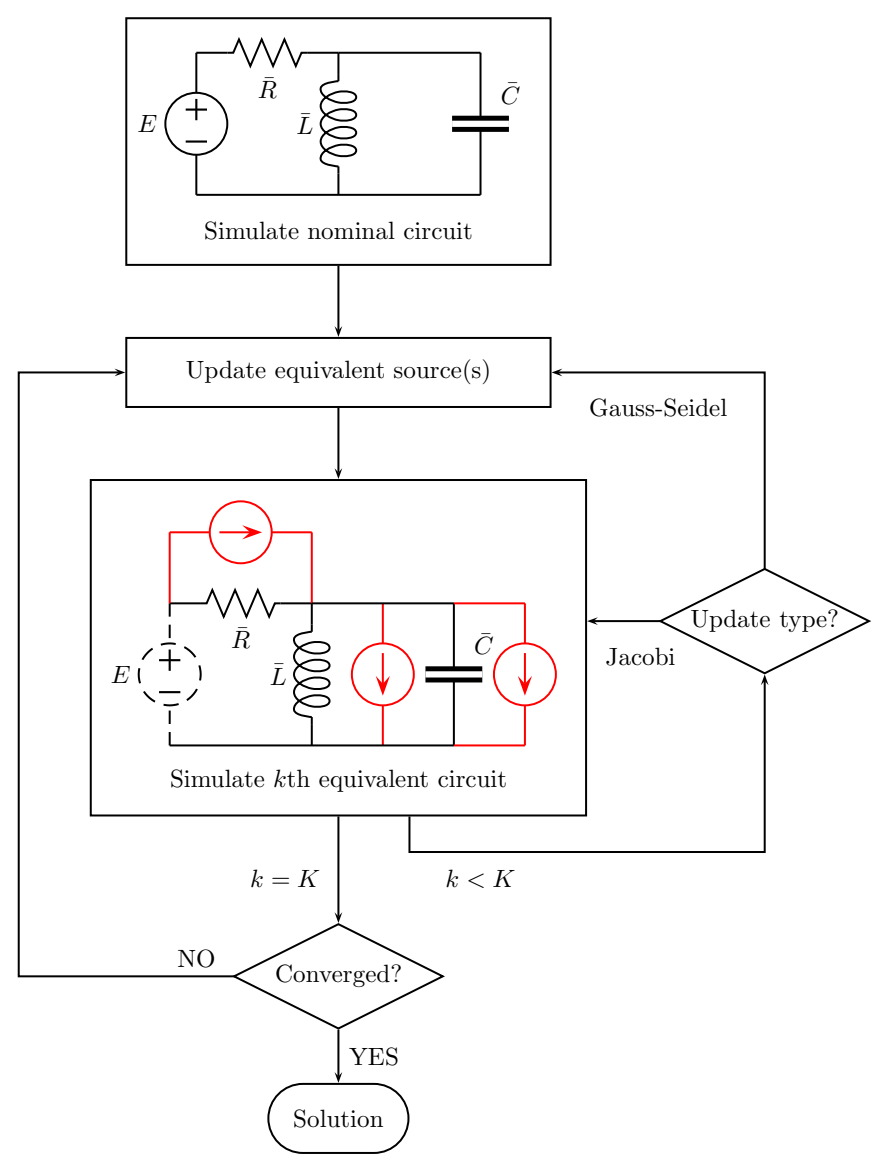

Fig. 2. Flowchart of the proposed perturbative SG simulation strategy.

loop over $k$ or once at the beginning of the loop, respectively. The process is repeated until convergence is reached. If the sparse implementation is adopted, starting from the second iteration, the circuit is re-simulated only if the corresponding PCE coefficient has been identified as non-negligible. It is important to point out that the proposed method retains the high accuracy of the SG method up to the selected tolerance for convergence. Furthermore, despite the presence of the additional equivalent sources, the circuit complexity remains unaltered in terms of nodal equations.

\section{E. Illustrative Example: Stochastic Resistor}

For the sake of illustration, the trivial case of a stochastic resistor subject to a constant voltage is discussed. The resistance value is assumed to be normally distributed with a mean value of $14 \Omega$ and a standard deviation of $2 \Omega$. The voltage source is $E=20 \mathrm{~V}$. With the above parameters, the resistor current $i=E / R(\xi)$ has a mean value of $1.4598 \mathrm{~A}$ and a standard deviation of $0.2235 \mathrm{~A}$.

The value of the stochastic resistance is expressed as $R(\xi)=\bar{R}+\mathcal{R}(\xi)$, with $\bar{R}=14 \Omega$ and $\mathcal{R}(\xi)=2 \cdot \xi \Omega$, where $\xi$ is a standard normal random variable. The overall equivalent circuits for the perturbative SG solution with $k=1$ and $k>1$ are shown in Fig. 3. It is readily noted that, at each iteration, $i_{1}=E / \bar{R}+i_{e q, 1}$, whereas $i_{k}=i_{e q, k}$ for $k>1$. 


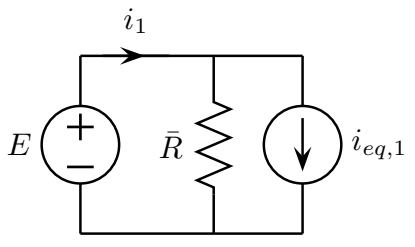

(a)

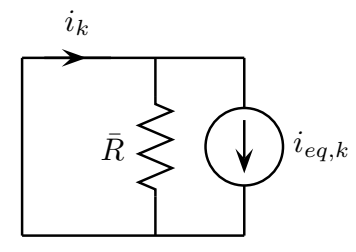

(b)
Fig. 3. Equivalent circuits for the perturbative calculation of the PCE coefficients with $k=1$ (a) and $k>1$ (b).

Using a second-order PCE with orthonormal Hermite polynomials

$$
\begin{aligned}
& \varphi_{1}=\phi_{0}=1 \\
& \varphi_{2}=\phi_{1}=\xi \\
& \varphi_{3}=\phi_{2}=\left(\xi^{2}-1\right) / \sqrt{2},
\end{aligned}
$$

the SG matrix for the update of the equivalent sources reads

$$
\widetilde{\mathcal{X}}=-\frac{1}{\bar{R}}\left(\begin{array}{lll}
\widetilde{\mathcal{R}}_{11} & \widetilde{\mathcal{R}}_{12} & \widetilde{\mathcal{R}}_{13} \\
\widetilde{\mathcal{R}}_{21} & \widetilde{\mathcal{R}}_{22} & \widetilde{\mathcal{R}}_{23} \\
\widetilde{\mathcal{R}}_{31} & \widetilde{\mathcal{R}}_{32} & \widetilde{\mathcal{R}}_{33}
\end{array}\right)=-\frac{2}{14}\left(\begin{array}{ccc}
0 & 1 & 0 \\
1 & 0 & \sqrt{2} \\
0 & \sqrt{2} & 0
\end{array}\right) .
$$

The starting nominal solution is $i_{1}=E / \bar{R}=1.4286$. Table I collects the values of the PCE coefficients of the resistor current and of the pertinent equivalent sources, obtained with both Jacobi and Gauss-Seidel updates, for iterations $m>0$. It is readily observed that the Gauss-Seidel solution achieves the same results as the Jacobi solution in about half of the iterations. After eight and five iterations, using Jacobi and Gauss-Seidel updates, respectively, the error on the mean value and standard deviation is below $0.0002 \%$ and $0.8 \%$, respectively. Obviously, for this simple one-dimensional case, all coefficients are significant and require updates.

For the sake of completeness, Fig. 4 shows the probability distribution of the resistor current. The empirical distribution obtained by MC sampling (gray bars) is in very good agreement with the PDF of the PCE model (red line). It is noted that the distribution is not Gaussian, but rather asymmetric because of the inverse relationship between the current and the resistance value.

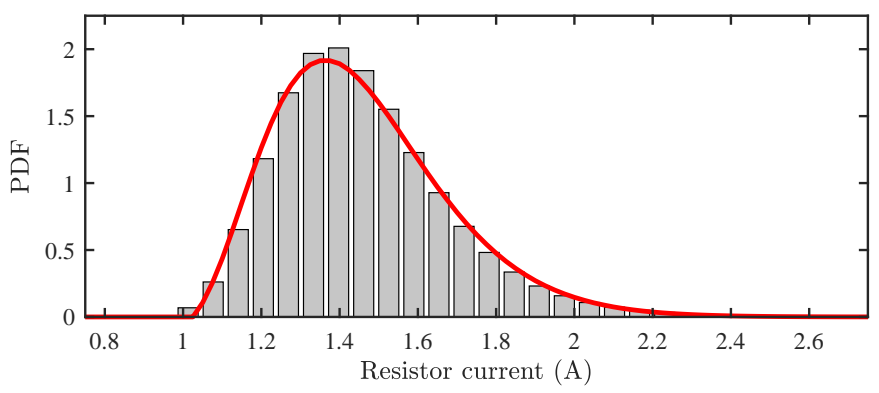

Fig. 4. Probability distribution of the resistor current obtained by MC sampling (gray bars) and by the PCE model (red line).

\section{IMPROVED SOLUTION FOR LTI CIRCUITS}

So far, the presented solution requires the equivalent sources to be updated iteratively, and the circuit re-simulated for each configuration thereof. When using a commercial circuit solver, such as SPICE or Simulink, equivalent waveforms can be supplied, e.g., in the form of piece-wise time-value pairs. The same approach is readily applied in the frequency domain, by using AC sources. With a customized solver, the probing of the necessary currents and the update of the equivalent sources could be carried out internally in a more efficient manner. However, a still more effective approach for LTI circuits is introduced in this section, taking advantage of the fact that the variability is in fact translated from components into sources, which are the only circuit elements changing throughout the iterations.

It is well-known that the response of a LTI system is fully characterized with respect to input signals in terms of impulse responses (or transfer functions in the frequency domain). Once the input-to-output impulse response (transfer function) is known, the response to an arbitrary excitation is obtained by a convolution (multiplication) between the two. As already noted, only the independent, i.e., the original and the equivalent, sources actually change throughout the iterations, whereas the rest of the circuit remains unchanged. These considerations allow introducing a further improvement in the solution strategy, in which the circuit is characterized in a single iteration in terms of the necessary responses in the nominal configuration. The update of the equivalent sources and of the corresponding PCE coefficients is calculated offline as post-processing.

\section{A. Time-Domain Analysis}

In the equivalent circuit for the perturbative SG solution (see, e.g., Fig. 2), the input signals that change throughout the iterations are both the original independent sources and the equivalent sources associated with stochastic components. The output signals that need to be computed are the original outputs of interest and the currents needed for the updates of the equivalent sources. Let us introduce an impulse-response matrix $h$ such that

$$
\left(\begin{array}{c}
\boldsymbol{i}_{\text {up }}(t) \\
\boldsymbol{x}_{\text {out }}(t)
\end{array}\right)=\boldsymbol{h}(t) *\left(\begin{array}{c}
\boldsymbol{u}_{\text {in }}(t) \\
\boldsymbol{i}_{\text {eq }}(t)
\end{array}\right)
$$

where:

- $\boldsymbol{i}_{\text {up }} \in \mathbb{R}^{d}$ collects the currents needed for the update of the equivalent sources for the $d$ stochastic elements;

- $\boldsymbol{x}_{\text {out }} \in \mathbb{R}^{n_{o}}$ collects $n_{o}$ voltage or current outputs of interest;

- $\boldsymbol{u}_{\text {in }} \in \mathbb{R}^{n_{i}}$ collects $n_{i}$ voltage or current excitations of the circuit;

- $\boldsymbol{i}_{e q} \in \mathbb{R}^{d}$ collects the equivalent current sources for the $d$ uncertain elements.

As such, $\boldsymbol{h} \in \mathbb{R}^{\left(d+n_{o}\right) \times\left(d+n_{i}\right)}$. The impulse-response matrix is effectively characterized column-wise, by calculating the step responses of the variables in the left-hand side of (29) in which all sources in the right-hand side are set to zero, except one that is set to a unitary value, and differentiating 
TABLE I

PERTURbative SG SOLUTION OF THE CURRENT OF A STOCHASTIC RESISTOR SUbJECT TO A CONSTANT VOLTAGE.

\begin{tabular}{|c|c|c|c|c|c|c|c|}
\hline \multirow[b]{2}{*}{ iteration $m$} & \multirow[b]{2}{*}{ PCE index $k$} & \multicolumn{3}{|c|}{ Jacobi update } & \multicolumn{3}{|c|}{ Gauss-Seidel update } \\
\hline & & equivalent source $i_{e q, k}$ & PCE coefficients $i_{k}$ & mean/std & equivalent source $i_{e q, k}$ & PCE coefficients $i_{k}$ & mean/std \\
\hline \multirow[t]{3}{*}{1} & 1 & 0 & 1.4286 & 1.4286 & 0 & 1.4286 & 1.4286 \\
\hline & 2 & -0.2041 & -0.2041 & 0.2041 & -0.2041 & -0.2041 & 0.2082 \\
\hline & 3 & 0 & 0 & & 0.0412 & 0.0412 & \\
\hline \multirow[t]{3}{*}{2} & 1 & 0.0292 & 1.4577 & 1.4577 & 0.0292 & 1.4577 & 1.4577 \\
\hline & 2 & -0.2041 & -0.2041 & 0.2082 & -0.2166 & -0.2166 & 0.2210 \\
\hline & 3 & 0.0412 & 0.0412 & & 0.0438 & 0.0438 & \\
\hline \multirow[t]{3}{*}{3} & 1 & 0.0292 & 1.4577 & 1.4577 & 0.0309 & 1.4595 & 1.4595 \\
\hline & 2 & -0.2166 & -0.2166 & 0.2205 & -0.2173 & -0.2173 & 0.2217 \\
\hline & 3 & 0.0412 & 0.0412 & & 0.0439 & 0.0439 & \\
\hline \multirow[t]{3}{*}{4} & 1 & 0.0309 & 1.4595 & 1.4595 & 0.0310 & 1.4596 & 1.4596 \\
\hline & 2 & -0.2166 & -0.2166 & 0.2210 & -0.2174 & -0.2174 & 0.2218 \\
\hline & 3 & 0.0438 & 0.0438 & & 0.0439 & 0.0439 & \\
\hline \multirow[t]{3}{*}{5} & 1 & 0.0309 & 1.4595 & 1.4595 & 0.0311 & 1.4596 & 1.4596 \\
\hline & 2 & -0.2173 & -0.2173 & 0.2217 & -0.2174 & -0.2174 & 0.2218 \\
\hline & 3 & 0.0438 & 0.0438 & & 0.0439 & 0.0439 & \\
\hline \multirow[t]{3}{*}{6} & 1 & 0.0310 & 1.4596 & 1.4596 & & & \\
\hline & 2 & -0.2173 & -0.2173 & 0.2217 & & & \\
\hline & 3 & 0.0439 & 0.0439 & & & & \\
\hline \multirow[t]{3}{*}{7} & 1 & 0.0310 & 1.4596 & 1.4596 & & & \\
\hline & 2 & -0.2174 & -0.2174 & 0.2218 & & & \\
\hline & 3 & 0.0439 & 0.0439 & & & & \\
\hline \multirow[t]{3}{*}{8} & 1 & 0.0311 & 1.4596 & 1.4596 & & & \\
\hline & 2 & -0.2174 & -0.2173 & 0.2218 & & & \\
\hline & 3 & 0.0439 & 0.0439 & & & & \\
\hline
\end{tabular}

the results. This only requires $d+n_{i}$ circuit simulations. Once the impulse-response matrix $\boldsymbol{h}$ is available, the SG iterations can be performed offline without further calls to the circuit simulator, and the equivalent sources, as well as the outputs of interests, are suitably updated by means of convolutions.

Remark. Since typically $d+n_{i} \ll K$, the proposed approach requires a number of actual circuit simulations that is by far smaller than any other state-of-the-art PCE-based approach.

\section{B. Frequency-Domain Analysis}

For AC analyses, a similar approach is adopted, in which the frequency-domain counterpart of the impulse-response matrix (29), i.e., the transfer function matrix $\boldsymbol{H}$, such that

$$
\left(\begin{array}{c}
\boldsymbol{I}_{u p}(f) \\
\boldsymbol{X}_{\text {out }}(f)
\end{array}\right)=\boldsymbol{H}(f) \cdot\left(\begin{array}{c}
\boldsymbol{U}_{i n}(f) \\
\boldsymbol{I}_{e q}(f)
\end{array}\right),
$$

is constructed. The iterative solution is carried out offline using mere matrix multiplications between $\boldsymbol{H}$ and the vector of independent sources.

\section{Illustrative Example: RLC Circuit}

As a first validation of the proposed simulation framework for LTI networks, the trivial RLC circuit of Fig. 2 is considered. The resistance, inductance, and capacitance are $d=3$ independent and normally distributed uncertain variables with nominal values $\bar{R}=10 \Omega, \bar{L}=200 \mathrm{nH}$, and $\bar{C}=1 \mathrm{nF}$, and a relative standard deviation of $10 \%$. The voltage source is $1 \mathrm{~V}$ and the output of interest is the voltage $v_{L C}$ across the LC resonator.
For the sake of comparison, the stochastic problem is simulated with:

1) the MC method;

2) the classical SG method [12];

3) the perturbative SG simulation for LTI circuits outlined in this section, with Jacobi or Gauss-Seidel updates.

The convergence of the perturbative iterations is checked based on the relative difference in norm- 2 between the matrices collecting the PCE coefficients from $k=2$ to $K$ for all simulated time or frequency points at two subsequent iterations. The first coefficient is excluded from the analysis because it is typically dominant over the others. For this and for the following examples, a threshold of $0.1 \%$ on the aforementioned metric is set to stop the iterations.

Figure 5 illustrates the variability of voltage $v_{L C}$ resulting from the uncertainty on the resistance, inductance, and capacitance values. The top panel shows a subset of samples from the MC analysis (gray lines). The voltage mean (top panel) and the standard deviation (bottom panel) computed from the MC samples and with the classical and perturbative SG techniques with second-order PCE (i.e., $p=2$ ) are also shown by solid blue, dashed red, and dotted green lines, respectively. Very good agreement is observed between all the curves for these two statistical moments. In particular, there is no appreciable difference between the results of the perturbative SG simulation with Jacobi and Gauss-Seidel updates. Therefore, only one curve is displayed in Fig. 5. Nevertheless, the two solutions require eight and five iterations to converge, respectively. Simulation times are irrelevant for such a trivial example, and therefore not discussed. In the 


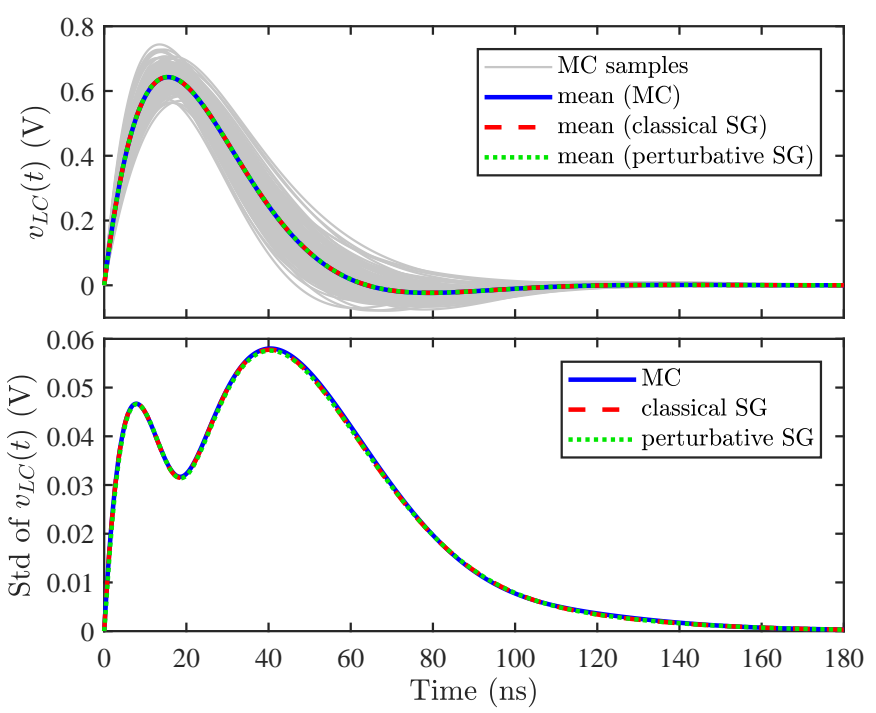

Fig. 5. Variability of voltage $v_{L C}$ in the RLC circuit of Fig. 2. Top panel: subset of MC samples (gray lines) and mean computed with the MC method (solid blue line), the classical SG method of [12] (dashed red line), and the proposed perturbative SG simulation (dotted green line). Bottom panel: standard deviation of voltage $v_{L C}$ computed with the same techniques.

following, the results of the perturbative SG simulation will be always displayed by means of a single curve, as both Jacobi and Gauss-Seidel solutions virtually converge to the same result within the specified tolerance.

\section{Vi. Application Examples and Results}

This section provides further and more significant validation examples involving various numbers of random circuit components. In this section, no distinction is made between the sparse and non-sparse implementations, as this choice mainly impacts efficiency, with negligible loss of accuracy. An extensive discussion on the performance of the proposed techniques is provided in the next section.

\section{A. Seventh-Order Chebyshev Low-Pass Filter}

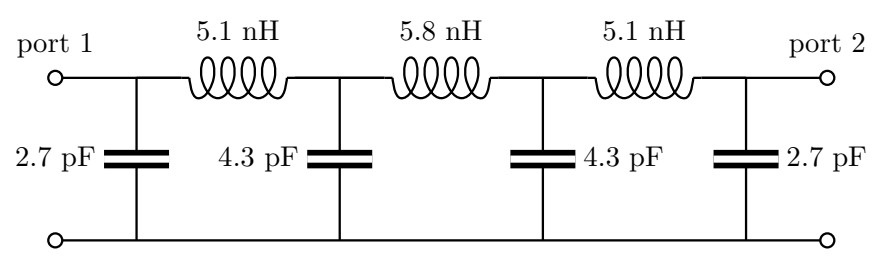

Fig. 6. Schematic of the seventh-order Chebyshev filter.

The first example concerns the seventh-order Chebyshev low-pass filter, illustrated in Fig. 6. The filter is designed to have a cut-off frequency of $2 \mathrm{GHz}$ and a passband ripple of $0.5 \mathrm{~dB}$. The component values indicated in Fig. 6 are the closest to the optimal design values available on the market. An independent Gaussian uncertainty with a relative standard deviation of $5 \%$ is ascribed to each element.

Fig. 7 shows the variability of the step response of the filter. In the top panel, the average response computed from the

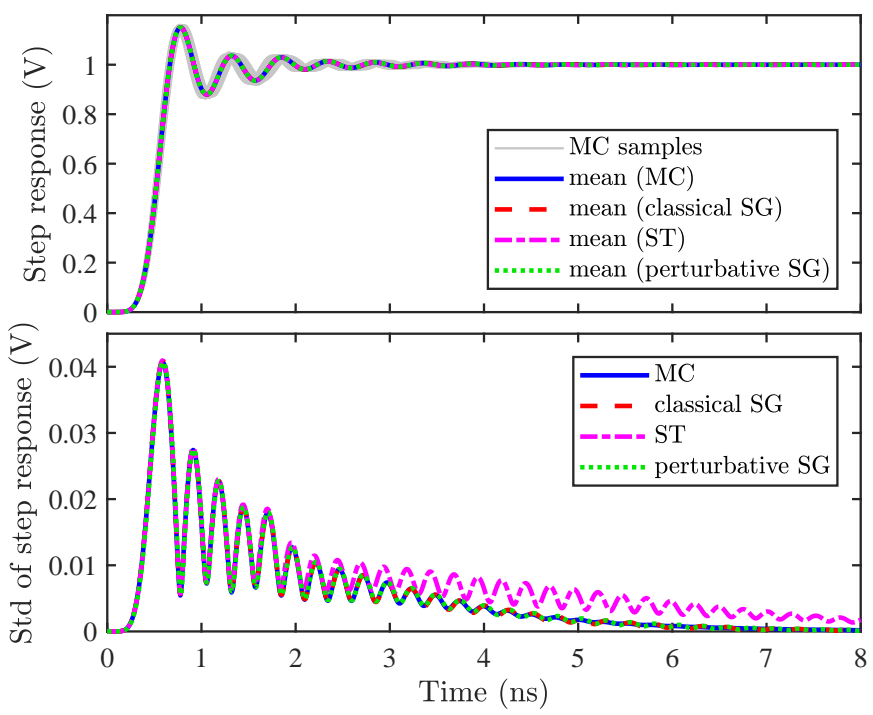

Fig. 7. Variability of the step response of the Chebyshev filter of Fig. 6. Top panel: subset of MC samples (gray lines) and mean computed with the MC method (solid blue line), the classical SG method [12] (dashed red line), the ST method [18] (dash-dotted magenta line), and the proposed perturbative SG simulation (dotted green line). Bottom panel: standard deviation of the step response computed with the same techniques.

MC samples, as well as with the classical SG method [12], with the interpolative ST method [18], and with the proposed perturbative SG simulation are superposed to a subset of MC samples. The bottom panel shows the standard deviation computed with the same stochastic techniques. Order $p=3$ is used for all PCE-based methods. While the Galerkin-based techniques provide accurate results, the ST method exhibits a large error on the standard deviation. This is probably due to the strong resonant behavior of the circuit, causing a large sensitivity of the responses with respect to the sampling points.

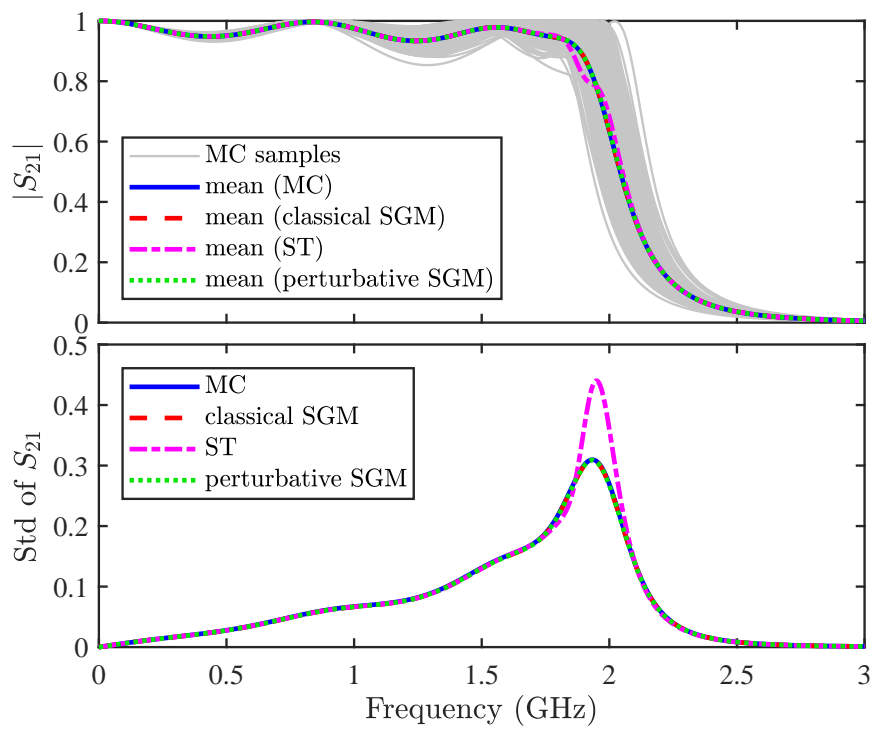

Fig. 8. Variability of the transmission $S_{21}$ of the Chebyshev filter.

Next, the analysis is repeated in the frequency domain. Figure 8 shows the uncertainty quantification of the magnitude 
of the filter transmission $S_{21}$. The inaccuracy of the ST method for this example is also confirmed in the frequency domain, since a large error is found around the cut-off frequency.

\section{B. Transmission-Line Network}

The second example considers a network with seven delay elements consisting of transmission-line sections of various lengths, as shown in Fig. 9. All relevant physical parameters are indicated in the figure. The voltage source produces a trapezoidal pulse with an amplitude of $1 \mathrm{~V}$, rise/fall times of $200 \mathrm{ps}$, and a width of $2.8 \mathrm{~ns}$ at half amplitude. The variability is provided by all the $d=29$ lumped components (a similar perturbative approach can be applied also to stochastic nonuniform transmission lines [34], but its implementation is not compatible - as is - with standard SPICE-type circuit simulators). To further assess the performance of the perturbative SG simulation for different amounts of variability, the element values are ascribed a Gaussian distribution with a relative standard deviation of increasing values, i.e., $3 \%, 5 \%$, $7 \%$, and $10 \%$.

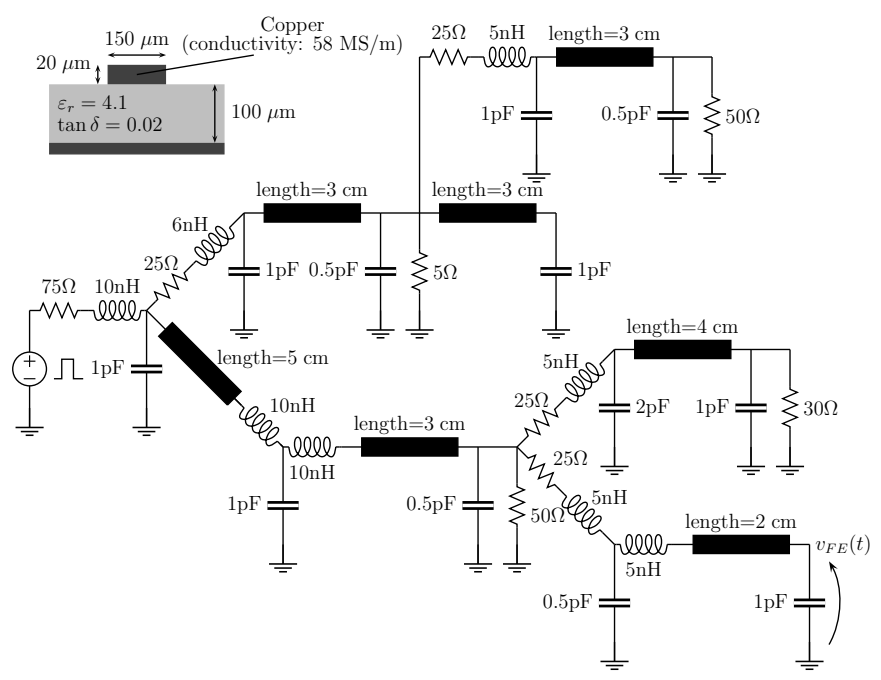

Fig. 9. Schematic of the network with transmission lines.

Figure 10 shows the voltage $v_{F E}$ transmitted to the far end of the network. In the top panel are a subset of samples from the MC simulation, highlighting the variability of the voltage resulting from the largest uncertainty in the lumped components (i.e., $10 \%$ standard deviation), as well as the corresponding average voltage computed from the MC samples and with the classical and perturbative SG simulations. The bottom panel provides the standard deviation of the voltage computed with the same three methods for the different standard deviations of the component values. Excellent agreement is found between the classical and the perturbative SG implementations for all cases, and their results also compare well with the MC references. As will be discussed in the next section, the amount of iterations required by the perturbative SG solution increases with the standard deviation of the component values. The ST method provides comparable results and is not shown in this analysis.
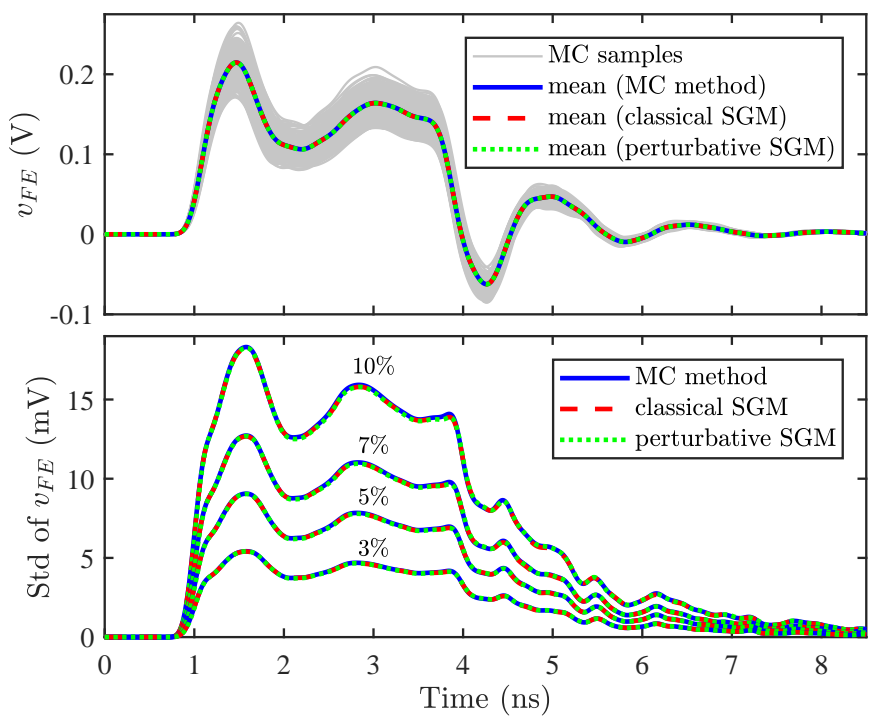

Fig. 10. Variability of voltage $v_{F E}$ in the network of Fig. 9. The top panel shows a subset of MC samples (gray lines) for the case with $10 \%$ variability. The mean obtained from the classical and perturbative SG simulations (dashed red and dotted green curves, respectively) is compared with the MC result (solid blue line). The bottom panel shows the standard deviations for different amounts of variability obtained with the same techniques.

Figure 11 shows the PDFs of voltage $v_{F E}$ at the time of maximum overshoot, i.e., $1.47 \mathrm{~ns}$, for the different amounts of uncertainty considered. The distribution of the MC samples (blue dots) is compared to the PDF estimated from the Galerkin-based simulations (solid red and dotted green lines), highlighting excellent agreement. Reasonably, the width of the PDF narrows as the uncertainty in the circuit parameters reduces.

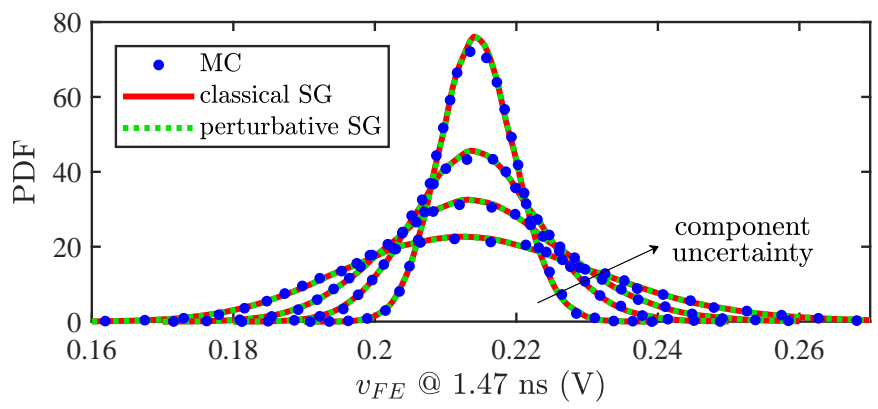

Fig. 11. Probability distribution of voltage $v_{F E}$ at $1.47 \mathrm{~ns}$ for different amounts of uncertainty. Blue dots: distribution of the MC samples; solid red and dotted green lines: predictions from the classical and perturbative SG simulations, respectively.

\section{Active Low-Pass Filter}

This test case concerns the active low-pass filter with the schematic of Fig. 12, reproduced from [33]. Without loss of generality, the opamps are considered ideal for simplicity. The variability is provided by the $d=30 \mathrm{RC}$ elements, which are ascribed independent Gaussian distributions with a standard deviation of 5\% from the nominal values indicated in Fig. 12. 


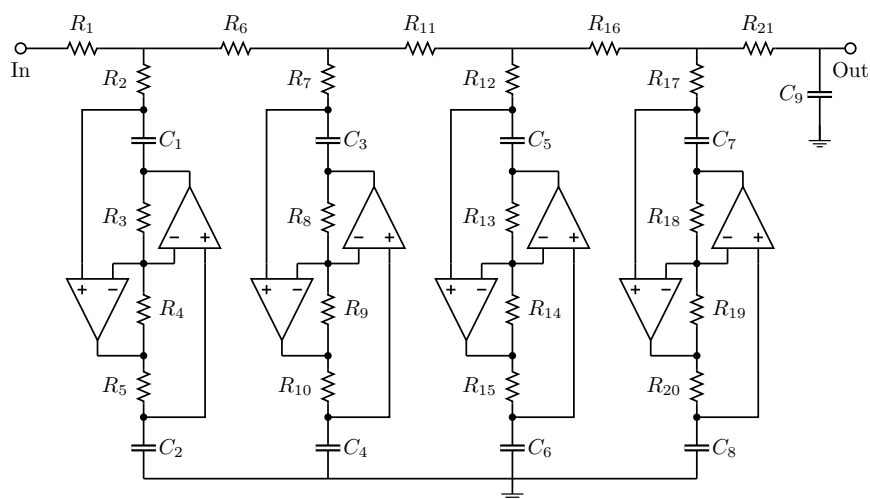

$\begin{array}{llll}R_{1}=5.4779 \mathrm{k} \Omega & R_{2}=2.0076 \mathrm{k} \Omega & R_{3,4,8,9,13,14,18,19}=3.3 \mathrm{k} \Omega & R_{5}=4.5898 \mathrm{k} \Omega\end{array}$ $\begin{array}{lllll}R_{6}=4.44 \mathrm{k} \Omega & R_{7}=5.9999 \mathrm{k} \Omega & R_{10}=4.2573 \mathrm{k} \Omega & R_{11}=3.2201 \mathrm{k} \Omega & R_{12}=5.88327 \mathrm{k} \Omega\end{array}$

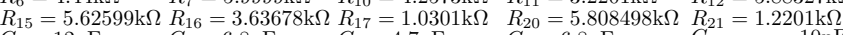
$\begin{array}{lllll}C_{1}=12 \mathrm{nF} & C_{3}=6.8 \mathrm{nF} & C_{5}=4.7 \mathrm{nF} & C_{7}=6.8 \mathrm{nF} & C_{2,4,6,8,9}=10 \mathrm{nF}\end{array}$

Fig. 12. Schematic of the active low-pass filter (reproduced from [33]).

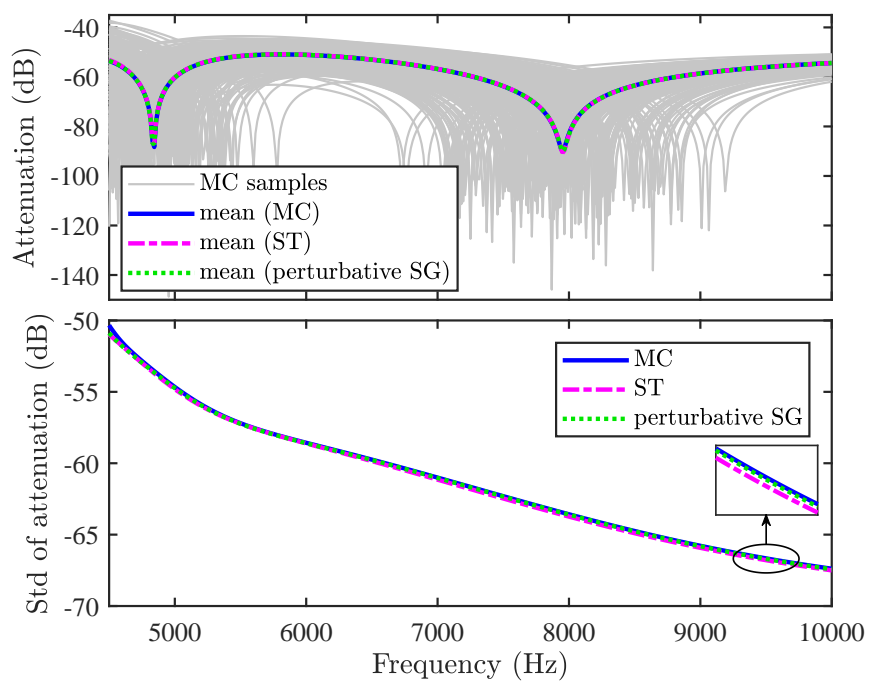

Fig. 13. Variability of the attenuation of the active filter of Fig. 12. Top panel: subset of samples from MC simulation (gray lines) and average attenuation estimated from the MC samples (solid blue line), with the ST method (dash-dotted magenta line), and with the proposed SG method (dotted green line). Bottom panel: standard deviation estimated with the same stochastic techniques.

Fig. 13 shows the variability of the filter input-to-output attenuation in the stopband. As in the previous test cases, the top panel shows a subset of random responses from the MC simulation (gray lines), as well as the average response. The result from the $\mathrm{MC}$ analysis (solid blue line) is compared to the one obtained with the ST method (dash-dotted magenta line) and the proposed SG simulation (dotted green line), both with order $p=2$. A huge variation of the attenuation and, especially, of the location of the dips is observed, despite the moderate uncertainty of the circuit components. Nonetheless, the PCE-based techniques provide an accurate estimation, also of the standard deviation (bottom panel). The perturbative SG result matches the reference MC curve better than the ST method at high frequencies, as shown by the inset. For this test case, the application of the state-of-the-art SG implementation of [12] was hindered by the difficulty in handling the ideal operational amplifiers. This reveals an additional advantage of the proposed perturbative technique, as no specific models are required for non-stochastic components.

\section{Full-Bridge Single-Phase Inverter}

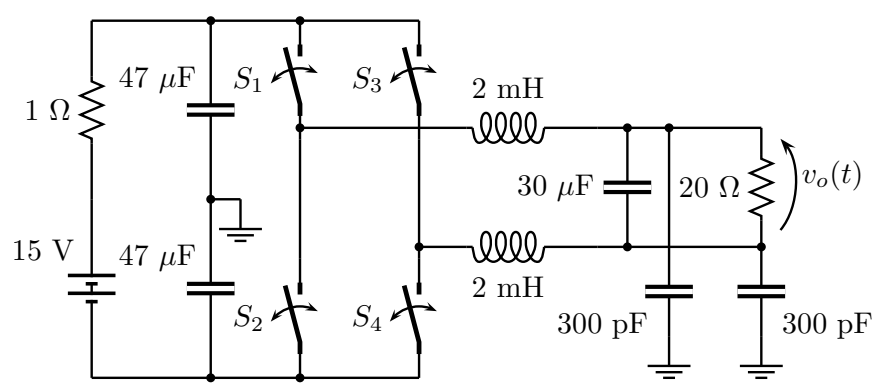

Fig. 14. Schematic of the full-bridge single-phase inverter.

The last example concerns the full-bridge single-phase inverter [35] shown in Fig. 14. This circuit is a DC-AC converter producing a $60-\mathrm{Hz}$ sinusoidal voltage from a constant input voltage. This is achieved by means of four switches with pulsewidth modulated controlling signals [36]. The variability is provided by all $d=9$ lumped components in the circuit, with a Gaussian distribution and a 15\% standard deviation. Whereas for all previous examples, the techniques of Section V were used, it should be noted that this inverter is a non-LTI circuit, for which the slower implementation of Section IV has to be applied. The system is simulated in Simulink. For the perturbative SG simulation, the updated equivalent sources are provided in the form of time-value pairs.

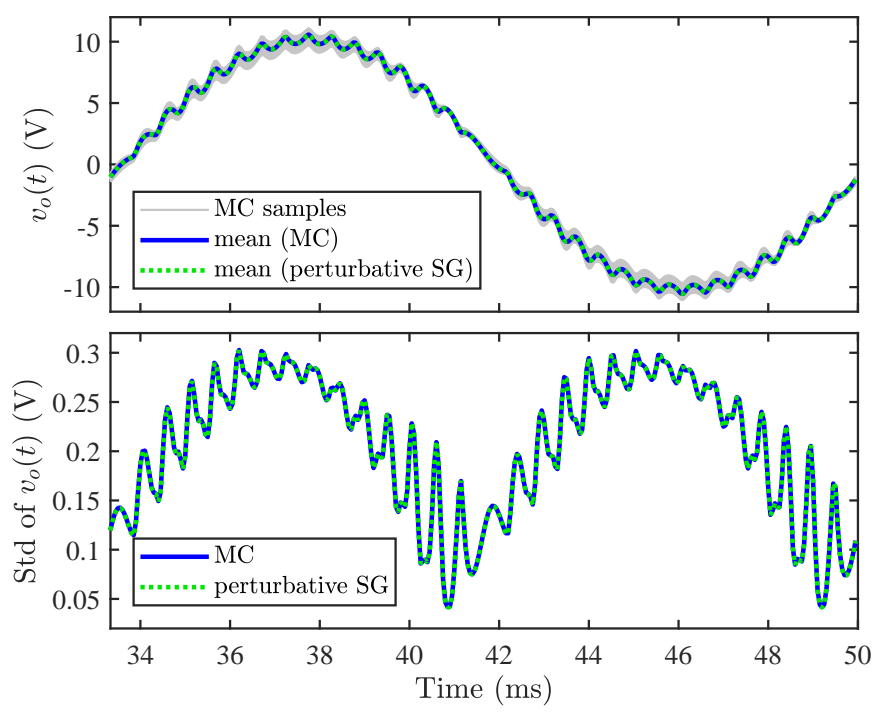

Fig. 15. Variability of the output voltage $v_{o}$ in the network of Fig. 14. Mean and standard deviation predicted with the proposed perturbative SG simulation are compared against the results from MC analysis (solid blue lines).

Fig. 15 shows the variability of the sinusoidal output voltage $v_{o}$. It is noted that the large variability has little impact on the output, and mainly affects the voltage ripple. The top panel shows a subset of samples from the MC simulation (gray lines) 
and the mean voltage obtained from the MC samples (solid blue line) and with the proposed perturbative SG simulation (dotted green line). The standard deviation obtained with these two techniques is provided in the bottom panel. The results obtained with the perturbative SG method match well with the MC estimates. The classical SG simulation of [12] is hardly applicable to this example and would require the development of models compatible with Simulink.

The main purpose of this example is to show the feasibility of the proposed perturbative SG reformulation for the simulation of non-LTI circuits in non-SPICE environments. However, the necessity of directly simulating the effect of the perturbative sources during each iteration in this case penalizes the performance with respect to sampling-based techniques such as the ST method.

\section{Computational EFFiciency AND Discussion}

The previous section discussed several application examples in both frequency and time domain, and focused on the accuracy of the various methods. Their efficiency is assessed in this section. All simulations are performed on a Dell Precision 5820 workstation with an Intel(R) Core(TM) i97900X, CPU running at $3.30 \mathrm{GHz}$ and $32 \mathrm{~GB}$ of RAM. For a fair comparison on the computational times for the perturbative SG simulation of LTI examples, involving post-processing convolutions over time or transfer function multiplications over frequency, the same number of time or frequency points is used, i.e., 1001 and 401, respectively.

The pertinent computational times are summarized in Table II. The purpose of the table is to compare the performance of the proposed perturbative SG simulation, and in particular of its sparse implementation, with respect to state-of-the-art PCE-based techniques. It was already widely proven that such methods outperform MC analyses, therefore a comparison in this regard is out of the scope of this paper. Here, MC is only used as a reference for the accuracy. However, as a brief example, it is worth mentioning that, for the network of Fig. 9, the generation of $10000 \mathrm{MC}$ samples for the reference results took 7646 s, i.e., nearly $10 \times$ slower than the slowest PCEbased technique.

First of all, it is interesting to note that, in spite of the larger number of iterations required, the Jacobi update strategy turns out to be more efficient than the non-sparse Gauss-Seidel one in most cases. This is explained by the fact that Jacobi updates allow performing post-processing operations simultaneously for all PCE coefficients, which is usually more effective than handling each coefficient separately within a loop. For frequency-domain simulations, the best performance of Jacobi updates is achieved by performing calculations separately for each frequency point, thus allowing the use of a different number of iterations ${ }^{1}$, while no significant difference is found for Gauss-Seidel updates in this regard. However, for the network of Fig. 9 the simulation with Jacobi updates incurs memory issues due to the very large size of the matrices to be handled for the simultaneous evaluation of the convolutions. Hence,

\footnotetext{
${ }^{1}$ The figure in the fourth column of the table indicates the highest iteration number in this case.
}

the Gauss-Seidel update, performing calculations separately for each PCE coefficient, turns out to be a viable solution for large-size problems. Moreover, the Gauss-Seidel strategy performs also better for the circuit of Fig. 14, which involves the direct simulation of the perturbative iterations, and benefits from the lower number of iterations required by this approach, rather than from post-processing efficiency.

When taking the sparsity of the PCE coefficients into account, the performance of Gauss-Seidel updates is further improved by avoiding the refinement of negligible coefficients. The sparsity index, indicated in the table, is defined as the ratio between the negligible PCE coefficients, based on the condition (28), and the total number of coefficients $K$. In most cases, the sparsity index identified after the first iteration matches the one obtained a posteriori from the standard SG simulation, based on the same criterion (28). Intuitively, the sparsity index increases when increasing the number of random parameters and/or reducing their variability (cfr. the transmission-line network example). It is interesting to note that the sparse implementation sometimes leads to a lower number of iterations, suggesting that the refinement of small coefficients may require additional iterations. As a result, the performance of the sparse algorithm scales better with the variability of input parameters.

Compared to the state-of-the-art SG implementation, the proposed perturbative SG simulation is more efficient when the number of random parameters is high, e.g., for the network of Fig. 9. It is important to remark that the circuit modification required to perform the perturbative SG simulation is minimal compared to the complex models required by the SG simulation in [12], and that the latter is not easily applicable to complex circuits. Indeed, the test cases of Fig. 12 and Fig. 14 could not be simulated with the state-of-the-art technique. Thanks to the efficient post-processing, the perturbative SG approach is also more efficient than the ST method for LTI circuits and usually provides superior accuracy.

It is also noted (see the transmission-line network example) that the simulation time for the perturbative SG method increases with the standard deviation of the component variability. This is expected, because a larger uncertainty leads to a higher number of iterations required for the method to converge. Therefore, the efficiency of the perturbative SG solution further increases as the variability reduces.

\section{CONCLUSIONS AND FUTURE WORK}

This paper presented a perturbative SG method for the simulation of stochastic linear circuits. The approach is based on interpreting component uncertainty as a perturbation from the nominal value. The application of the SG method to relaxed governing equations leads to decoupled equations for the pertinent PCE coefficients. Such equations are equivalent to a component with its nominal value paralleled by a current source. This equivalent circuit is readily implementable in any circuit solver. The solution is calculated iteratively by updating the equivalent current sources using Jacobi or GaussSeidel strategies, with the latter usually halving the number of required iterations. However, for LTI circuits, the circuit can 
TABLE II

COMPUTATIONAL TIMES OF THE VARIOUS PCE-BASED TECHNIQUES FOR THE CONSIDERED APPLICATION TEST CASES.

\begin{tabular}{|c|c|c|c|c|c|}
\hline Test case & $\bar{d}$ & Method & Iteration \# & Time & Speed-up \\
\hline $\begin{array}{l}\text { Chebyshev filter } \\
\text { (time domain) }\end{array}$ & 7 & $\begin{array}{l}\text { classical SG } \\
\text { ST } \\
\text { perturbative SG (Jacobi updates) } \\
\text { perturbative SG (Gauss-Seidel updates) } \\
\text { sparse perturbative SG (Gauss-Seidel updates, sparsity index }=80.8 \% \text { ) }\end{array}$ & $\begin{array}{c}13 \\
7 \\
6\end{array}$ & $\begin{array}{r}3.0 \mathrm{~s} \\
10.5 \mathrm{~s} \\
5.1 \mathrm{~s} \\
8.3 \mathrm{~s} \\
3.3 \mathrm{~s}\end{array}$ & $\begin{array}{l}- \\
- \\
- \\
-\end{array}$ \\
\hline $\begin{array}{l}\text { Chebyshev filter } \\
\text { (frequency domain) }\end{array}$ & 7 & $\begin{array}{l}\text { classical SG } \\
\text { ST } \\
\text { perturbative SG (Jacobi updates) } \\
\text { perturbative SG (Gauss-Seidel updates) } \\
\text { sparse perturbative SG (Gauss-Seidel updates, sparsity index }=9.2 \% \text { ) }\end{array}$ & $\begin{array}{l}22 \\
12 \\
12\end{array}$ & $\begin{array}{l}0.4 \mathrm{~s} \\
9.5 \mathrm{~s} \\
1.2 \mathrm{~s} \\
2.7 \mathrm{~s} \\
2.3 \mathrm{~s}\end{array}$ & $\begin{array}{l}- \\
- \\
-\end{array}$ \\
\hline Transmission-line network & 29 & $\begin{array}{l}\text { classical SG } \\
\text { ST } \\
\text { perturbative SG (Jacobi updates) } \\
\text { perturbative SG (Gauss-Seidel updates) } \\
\quad \text { variability }=3 \% \\
\quad \text { variability }=5 \% \\
\text { variability }=7 \% \\
\text { variability }=10 \% \\
\text { sparse perturbative SG (Gauss-Seidel updates) } \\
\quad \text { variability }=3 \%, \text { sparsity index }=95.5 \% \\
\quad \text { variability }=5 \%, \text { sparsity index }=95.1 \% \\
\text { variability }=7 \%, \text { sparsity index }=93.8 \% \\
\text { variability }=10 \%, \text { sparsity index }=88.0 \%\end{array}$ & $\begin{array}{c}3 \\
4 \\
9 \\
17 \\
\\
3 \\
3 \\
5 \\
9\end{array}$ & $\begin{array}{r}437 \mathrm{~s} \\
257 \mathrm{~s} \\
\mathrm{n} / \mathrm{a} \\
152 \mathrm{~s} \\
198 \mathrm{~s} \\
430 \mathrm{~s} \\
803 \mathrm{~s} \\
66 \mathrm{~s} \\
65 \mathrm{~s} \\
72 \mathrm{~s} \\
107 \mathrm{~s}\end{array}$ & $\begin{array}{r}2.9 \times \\
2.2 \times \\
1.0 \times \\
- \\
6.6 \times \\
6.7 \times \\
6.1 \times \\
4.1 \times\end{array}$ \\
\hline Active low-pass filter & 30 & $\begin{array}{l}\text { classical SG } \\
\text { ST } \\
\text { perturbative SG (Jacobi updates) } \\
\text { perturbative SG (Gauss-Seidel updates) } \\
\text { sparse perturbative SG (Gauss-Seidel updates, sparsity index }=53.6 \% \text { ) }\end{array}$ & $\begin{array}{l}9 \\
4 \\
4\end{array}$ & $\begin{array}{r}\mathrm{n} / \mathrm{a} \\
38.6 \mathrm{~s} \\
4.8 \mathrm{~s} \\
97.5 \mathrm{~s} \\
60.0 \mathrm{~s}\end{array}$ & $\begin{array}{l}\mathrm{n} / \mathrm{a} \\
\mathrm{n} / \mathrm{a} \\
\mathrm{n} / \mathrm{a} \\
\mathrm{n} / \mathrm{a}\end{array}$ \\
\hline Inverter & 9 & $\begin{array}{l}\text { classical SG } \\
\text { ST } \\
\text { perturbative SG (Jacobi updates) } \\
\text { perturbative SG (Gauss-Seidel updates) } \\
\text { sparse perturbative SG (Gauss-Seidel updates, sparsity index }=72.7 \% \text { ) }\end{array}$ & $\begin{array}{l}6 \\
4 \\
4\end{array}$ & $\begin{array}{r}\mathrm{n} / \mathrm{a} \\
66 \mathrm{~s} \\
1484 \mathrm{~s} \\
994 \mathrm{~s} \\
476 \mathrm{~s}\end{array}$ & $\begin{array}{l}\mathrm{n} / \mathrm{a} \\
\mathrm{n} / \mathrm{a} \\
\mathrm{n} / \mathrm{a} \\
\mathrm{n} / \mathrm{a}\end{array}$ \\
\hline
\end{tabular}

be effectively characterized by means of impulse responses or transfer functions, and the iterations carried out in postprocessing. In this scenario, the slower convergence of Jacobi iterations is offset by their superior computational efficiency, except when matrix dimensions make the calculations intractable or a sparse implementation is adopted, thus avoiding an unnecessary refinement of negligible coefficients.

The proposed perturbative method scales better with the number of stochastic dimensions than the state-of-the-art implementations of the SG method. Moreover, the method exhibits an accuracy that is comparable to the classical SG implementation, and thus often superior to the one of the interpolative ST method. Since it requires very little modification to the circuit, and only to its stochastic components, the method allows the direct use of SPICE-type circuit simulators and readily applies to arbitrary linear circuits, including networks that cannot be handled with the models in [12].

Plans for future work include improving the convergence of the iterations and the calculation of time-domain convolutions, as well as extension to nonlinear circuits. To this end, a combination with waveform relaxation approaches [37], which allow decoupling and separately solving the linear and nonlinear parts of a circuit, seems to be a promising strategy.

\section{REFERENCES}

[1] R. Spence and R. S. Soin, Tolerance Design of Electronic Circuits. London: Imperial College Press, 1997.

[2] A. Kaintura, T. Dhaene, and D. Spina, "Review of polynomial chaosbased methods for uncertainty quantification in modern integrated circuits," Electronics, vol. 7, no. 3, p. 30:1-21, Feb. 2018.

[3] D. Xiu and G. E. Karniadakis, "The Wiener-Askey polynomial chaos for stochastic differential equations," SIAM J. Sci. Computation, vol. 24, no. 2, pp. 619-622, 2002.

[4] D. Xiu, "Fast numerical methods for stochastic computations: a review," Commun. Computational Physics, vol. 5, no. 2-4, pp. 242-272, Feb. 2009.

[5] R. G. Ghanen and P. D. Spanos, Stochastic Finite Elements. A Spectral Approach. New York: Springer-Verlag, 1991.

[6] J. Bai, G. Zhang, D. Wang, A. P. Duffy, and L. Wang, "Performance comparison of the SGM and the SCM in EMC Simulation," IEEE Trans. Electromagn. Compat., vol. 58, no. 6, pp. 1739-1746, Dec. 2016.

[7] S. Vrudhula, J. M. Wang, and P. Ghanta, "Hermite polynomial based interconnect analysis in the presence of process variations," IEEE Trans. Comput. Aided Design Int. Circ. Syst., vol. 25, no. 10, pp. 20012011, Oct. 2006. 
[8] N. Mi, S. X.-D. Tan, Y. Cai, and X. Hong, "Fast variational analysis of on-chip power grids by stochastic extended Krylov subspace method," IEEE Trans. Comput. Aided Design Int. Circ. Syst., vol. 27, no. 11, pp. 1996-2006, Nov. 2008.

[9] Q. Su and K. Strunz, "Stochastic circuit modelling with Hermite polynomial chaos," IET Electronics Letters, vol. 41, no. 21, pp. 11631165 , Oct. 2005.

[10] K. Strunz and Q. Su, "Stochastic formulation of SPICE-type electronic circuit simulation using polynomial chaos," ACM Trans. Model. Comput. Simul., vol. 18, no. 4, pp. 15:1-15:23, Sep. 2008.

[11] P. Manfredi, D. Vande Ginste, D. De Zutter, and F. G. Canavero, "Uncertainty assessment of lossy and dispersive lines in SPICE-type environments," IEEE Trans. Compon. Packag. Manuf. Techol., vol. 3, no. 7, pp. 1252-1258, Jul. 2013.

[12] P. Manfredi, D. Vande Ginste, D. De Zutter, and F. G. Canavero, "Stochastic modeling of nonlinear circuits via SPICE-compatible spectral equivalents," IEEE Trans. Circuits Syst. I, Reg. Papers, vol. 61, no. 7, pp. 2057-2065, Jul. 2014

[13] P. Manfredi, D. Vande Ginste, D. De Zutter, and F. G. Canavero, 'On the passivity of polynomial chaos-based augmented models for stochastic circuits," IEEE Trans. Circuits Syst. I, Reg. Papers, vol. 60, no. 11, pp. 2998-3007, Nov. 2013.

[14] P. Manfredi, A. Biondi, D. Vande Ginste, D. De Zutter, and F. G. Canavero, "SPICE-based statistical assessment of interconnects terminated by nonlinear loads with polynomial characteristics," in Proc. IEEE 22th Conf. Elect. Perform. Electron. Packag. Syst., San Jose, CA, USA, Oct.2013, pp. 99-102.

[15] M. R. Rufuie, E. Gad, M. Nakhla, and R. Achar, "Generalized Hermite polynomial chaos for variability analysis of macromodels embedded in nonlinear circuits," IEEE Trans. Compon. Packag. Manuf. Technol., vol. 4, no. 4, pp. 673-684, Apr. 2014.

[16] P. Manfredi and F. Canavero, "Efficient statistical simulation of microwave devices via stochastic testing-based circuit equivalents of nonlinear components," IEEE Trans. Microw. Theory Techn., vol. 63, no. 5, pp. 1502-1511, May 2015.

[17] Z. Zhang, T. A. El-Moselhy, I. M. Elfadel, and L. Daniel, "Stochastic testing method for transistor-level uncertainty quantification based on generalized polynomial chaos," IEEE Trans. Comput.-Aided Des. Integr. Circuits Syst., vol. 32, no. 10, pp. 1533-1545, Oct. 2013.

[18] P. Manfredi, D. Vande Ginste, D. De Zutter, and F. G. Canavero, "Generalized decoupled polynomial chaos for nonlinear circuits with many random parameters," IEEE Microw. Wireless Compon. Lett., vol. 25, no. 8, pp. 505-507, Aug. 2015.

[19] M. Ahadi and S. Roy, "Sparse linear regression (SPLINER) approach for efficient multidimensional uncertainty quantification of high-speed circuits," IEEE Trans. Comput.-Aided Des. Integr. Circuits Syst., vol. 35, no. 10, pp. 1640-1652, Oct. 2016.

[20] D. Spina, F. Ferranti, T. Dhaene, L. Knockaert, and G. Antonini, "Polynomial chaos-based macromodeling of multiport systems using an input-output approach," Int. J. Numer. Model., vol. 28, no. 5, pp. 562 581, 2015.

[21] A. K. Prasad, M. Ahadi, and S. Roy, "Multidimensional uncertainty quantification of microwave/RF networks using linear regression and optimal design of experiments," IEEE Trans. Microw. Theory Techn. vol. 64, no. 8, pp. 2433-2446, Aug. 2016.

[22] Z. Zhang, T. Weng, and L. Daniel, "Big-data tensor recovery for high-dimensional uncertainty quantification of process variations," IEEE Trans. Compon. Packag. Manuf. Technol., vol. 7, no. 5, pp. 687-697, May 2017.

[23] P. Manfredi, R. Trinchero, and D. Vande Ginste, "Variability analysis of a boost converter based on an iterative and decoupled circuit implementation of the stochastic Galerkin method," in Proc. of the 2018 Joint IEEE Int. Symp. Electromagn. Compat. \& Asia-Pacific Symp. Electromagn. Compat., Singapore, May 2018, pp. 996-1000.

[24] C. Cui and Z. Zhang, "Stochastic collocation with non-Gaussian correlated process variations: theory, algorithms and applications," IEEE Trans. Compon. Packag. Manuf. Technol., vol. 9, no. 7, pp. 1362-1375, Jul. 2019.

[25] C. Cui and Z. Zhang, "High-dimensional uncertainty quantification of electronic and photonic IC with non-Gaussian correlated process variations," IEEE Trans. Comput.-Aided Des. Integr. Circuits Syst. (early access), DOI: 10.1109/TCAD.2019.2925340

[26] D. Cox, J. Little, and D. O'Shea, Ideals, Varieties, and Algorithms: An Introduction to Computational Algebraic Geometry and Commutative Algebra. 3rd ed. New York: Springer, 2007.

[27] P. Manfredi, D. Vande Ginste, I. S. Stievano, D. De Zutter, and F. G. Canavero, "Stochastic transmission line analysis via polynomial chaos methods: an overview," IEEE Electromagn. Compat. Mag., vol. 6, no. 3, pp. 77-84, 2017.

[28] P. Manfredi, D. De Zutter, and D. Vande Ginste, "On the relationship between the stochastic Galerkin method and the pseudo-spectral collocation method for linear differential algebraic equations," J. Eng. Math., vol. 108, no. 1, pp.73-90, Feb. 2018.

[29] D. M. Young, Iterative Solution of Large Linear Systems. Orlando: Academic Press, 1971.

[30] G. Blatman and B. Sudret, "An adaptive algorithm to build up sparse polynomial chaos expansions for stochastic finite element analysis," Probabilistic Eng. Mech., vol. 25, no. 2, pp. 183-197, Apr. 2010.

[31] G. Blatman and B. Sudret, "Adaptive sparse polynomial chaos expansion based on least angle regression," J. Comput. Phys., vol. 230, pp. 2345 2367, Mar. 2011

[32] J. Guo, Y. Xie, and F. Rachidi, "Modeling of EMP coupling to lossless MTLs in time domain based on analytical Gauss-Seidel iteration technique," in Proc. of the 2018 Joint IEEE Int. Symp. Electromagn. Compat. \& Asia-Pacific Symp. Electromagn. Compat., Singapore, May 2018, pp. 897-902.

[33] T.-A. Pham, E. Gad, M. S. Nakhla, and R. Achar, "Decoupled polynomial chaos and its applications to statistical analysis of high-speed interconnects," IEEE Trans. Compon. Packag. Manuf. Techol., vol. 4, no. 10, pp. 1634-1647, Oct. 2014.

[34] X. Wu, P. Manfredi, D. Vande Ginste, and F. Grassi, "A hybrid perturbative-stochastic Galerkin method for the variability analysis of nonuniform transmission lines," IEEE Trans. Electromagn. Compat. (early access), DOI 10.1109/TEMC.2019.2922407

[35] M. H. Rashid, Power Electronics: Devices, Circuits, and Applications. 4th ed. Harlow, UK: Pearson, 2014.

[36] R. Trinchero, P. Manfredi, I. S. Stievano, and F. G. Canavero, "Steadystate analysis of switching converters via frequency-domain circuit equivalents," IEEE Trans. Circuits Syst. II, Exp. Briefs, vol. 63, no. 8, pp. 748-752, Aug. 2016.

[37] V. Loggia, S. Grivet-Talocia, and $\mathrm{H}$. Hu, "Transient simulation of complex high-speed channels via waveform relaxation," IEEE Trans. Compon. Packag. Manuf. Techol., vol. 1, no. 11, pp. 1823-1838, Nov. 2011. 